NASA/TM-2013-216573

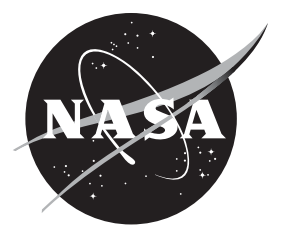

\title{
Analysis of Water Recovery Rate From the Heat Melt Compactor
}

R. Balasubramaniam and U. Hegde

National Center for Space Exploration Research, Glenn Research Center, Cleveland, Ohio

S. Gokoglu

Glenn Research Center, Cleveland, Ohio 


\section{NASA STI Program . . . in Profile}

Since its founding, NASA has been dedicated to the advancement of aeronautics and space science. The NASA Scientific and Technical Information (STI) program plays a key part in helping NASA maintain this important role.

The NASA STI Program operates under the auspices of the Agency Chief Information Officer. It collects, organizes, provides for archiving, and disseminates NASA's STI. The NASA STI program provides access to the NASA Aeronautics and Space Database and its public interface, the NASA Technical Reports Server, thus providing one of the largest collections of aeronautical and space science STI in the world. Results are published in both non-NASA channels and by NASA in the NASA STI Report Series, which includes the following report types:

- TECHNICAL PUBLICATION. Reports of completed research or a major significant phase of research that present the results of NASA programs and include extensive data or theoretical analysis. Includes compilations of significant scientific and technical data and information deemed to be of continuing reference value. NASA counterpart of peer-reviewed formal professional papers but has less stringent limitations on manuscript length and extent of graphic presentations.

- TECHNICAL MEMORANDUM. Scientific and technical findings that are preliminary or of specialized interest, e.g., quick release reports, working papers, and bibliographies that contain minimal annotation. Does not contain extensive analysis.

- CONTRACTOR REPORT. Scientific and technical findings by NASA-sponsored contractors and grantees.
- CONFERENCE PUBLICATION. Collected papers from scientific and technical conferences, symposia, seminars, or other meetings sponsored or cosponsored by NASA.

- SPECIAL PUBLICATION. Scientific, technical, or historical information from NASA programs, projects, and missions, often concerned with subjects having substantial public interest.

- TECHNICAL TRANSLATION. Englishlanguage translations of foreign scientific and technical material pertinent to NASA's mission.

Specialized services also include creating custom thesauri, building customized databases, organizing and publishing research results.

For more information about the NASA STI program, see the following:

- Access the NASA STI program home page at http://www.sti.nasa.gov

- E-mail your question to help@sti.nasa.gov

- Fax your question to the NASA STI Information Desk at 443-757-5803

- Phone the NASA STI Information Desk at 443-757-5802

- Write to: STI Information Desk NASA Center for AeroSpace Information 7115 Standard Drive Hanover, MD 21076-1320 
NASA/TM-2013-216573

AIAA-2013-3393

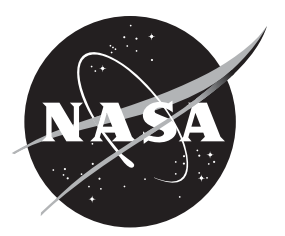

\section{Analysis of Water Recovery Rate From the Heat Melt Compactor}

\section{R. Balasubramaniam and U. Hegde}

National Center for Space Exploration Research, Glenn Research Center, Cleveland, Ohio

S. Gokoglu

Glenn Research Center, Cleveland, Ohio

Prepared for the

43rd International Conference on Environmental Systems

sponsored by the American Institute for Aeronautics and Astronautics

Vail, Colorado, July 14-18, 2013

National Aeronautics and

Space Administration

Glenn Research Center

Cleveland, Ohio 44135 


\section{Acknowledgments}

The authors gratefully acknowledge the support of this work by the Heat Melt Compactor project which is a part of the NASA Advanced Exploration Systems program. R. Balasubramaniam and U. Hegde were supported under NASA Contract No. NNC08BA08B with the National Center for Space Exploration Research. The authors thank G. Pace and J. Fisher of NASAAmes

Research Center and E. Golliher, D. Linne, K. Sacksteder and J. Lytle of NASA Glenn Research Center for technical discussions and insights during the course of this investigation.

Trade names and trademarks are used in this report for identification only. Their usage does not constitute an official endorsement, either expressed or implied, by the National Aeronautics and Space Administration.

Level of Review: This material has been technically reviewed by technical management.

Available from

NASA Center for Aerospace Information 7115 Standard Drive

Hanover, MD 21076-1320
National Technical Information Service 5301 Shawnee Road Alexandria, VA 22312

Available electronically at http://www.sti.nasa.gov 


\title{
Analysis of Water Recovery Rate From the Heat Melt Compactor
}

\author{
R. Balasubramaniam and U. Hegde \\ National Center for Space Exploration Research \\ Glenn Research Center \\ Cleveland, Ohio 44135 \\ S. Gokoglu \\ National Aeronautics and Space Administration \\ Glenn Research Center \\ Cleveland, Ohio 44135
}

\begin{abstract}
Human space missions generate trash with a substantial amount of plastic (20\% or greater by mass). The trash also contains water trapped in food residue and paper products and other trash items. The Heat Melt Compactor (HMC) under development by NASA Ames Research Center (ARC) compresses the waste, dries it to recover water and melts the plastic to encapsulate the compressed trash. The resulting waste disk or puck represents an approximately ten-fold reduction in the volume of the initial trash loaded into the HMC. In the current design concept being pursued, the trash is compressed by a piston after it is loaded into the trash chamber. The piston face, the side walls of the waste processing chamber and the end surface in contact with the waste can be heated to evaporate the water and to melt the plastic. Water is recovered by the HMC in two phases. The first is a pre-process compaction without heat or with the heaters initially turned on but before the waste heats up. Tests have shown that during this step some liquid water may be expelled from the chamber. This water is believed to be free water (i.e., not bound with or absorbed in other waste constituents) that is present in the trash. This phase is herein termed Phase A of the water recovery process. During HMC operations, it is desired that liquid water recovery in Phase A be eliminated or minimized so that water-vapor processing equipment (e.g., condensers) downstream of the HMC are not fouled by liquid water and its constituents (i.e., suspended or dissolved matter) exiting the HMC. The primary water recovery process takes place next where the trash is further compacted while the heated surfaces reach their set temperatures for this step. This step will be referred to herein as Phase B of the water recovery process. During this step the waste chamber may be exposed to different selected pressures such as ambient, low pressure (e.g., 0.2 atm), or vacuum. The objective for this step is to remove both bound and any remaining free water in the trash by evaporation. The temperature settings of the heated surfaces are usually kept above the saturation temperature of water but below the melting temperature of the plastic in the waste during this step to avoid any encapsulation of wet trash which would reduce the amount of recovered water by blocking the vapor escape. In this paper, we analyze the water recovery rate during Phase B where the trash is heated and water leaves the waste chamber as vapor, for operation of the HMC in reduced gravity. We pursue a quasi-one-dimensional model with and without sidewall heating to determine the water recovery rate and the trash drying time. The influences of the trash thermal properties, the amount of water loading, and the distribution of the water in the trash on the water recovery rates are determined.
\end{abstract}




\section{Nomenclature}

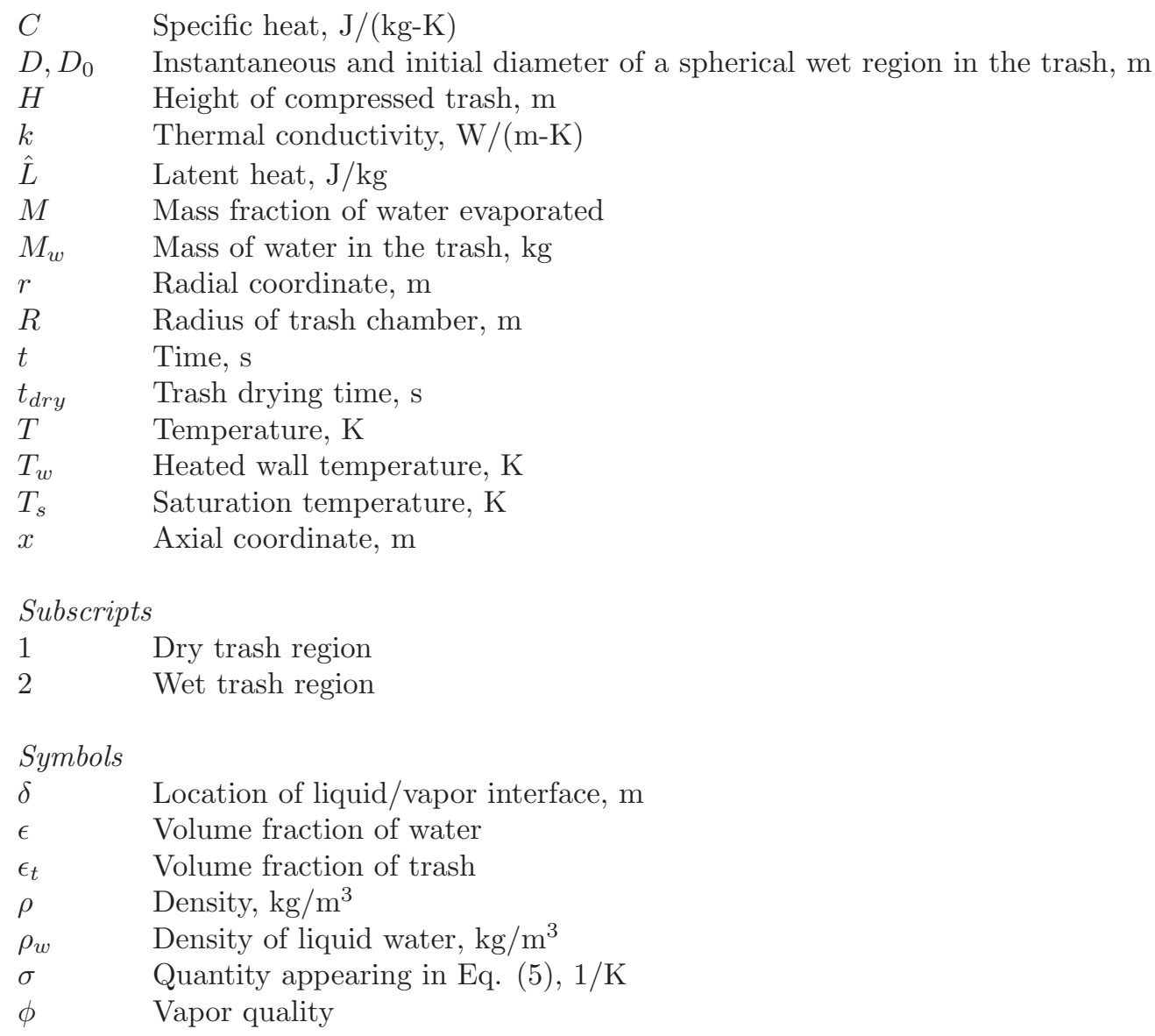

\section{Introduction}

Human space missions generally generate trash with a substantial amount of plastic ( $20 \%$ or greater by mass). The trash also contains water trapped in food residue and paper products and other trash items. The Heat Melt Compactor (HMC) under development by NASA Ames Research Center (ARC) compresses the waste, dries it to recover water, and melts the plastic to encapsulate the compressed trash. The resulting waste disk or puck represents an approximately ten-fold reduction in the volume of the initial trash loaded into the HMC.

Figure 1 shows a cut-away view of the initial design concept. The trash is loaded into the waste processing chamber where it is compressed by the piston. The piston face, the side walls of the waste processing chamber and also the surface in contact with the waste at the input door can be heated to first evaporate the water and second to melt the plastic. The major steps in the overall process are:

1. Trash loading into HMC.

2. Primary compaction without heat or with the heaters on but before the waste heats up. This phase is herein termed Phase A of the water recovery process. Typical compaction time is on the order of 2 minutes.

3. Primary water extraction with secondary compaction. This step will be referred to herein as Phase B of the water recovery process. Typical time is on the order of 80 minutes in initial tests.

4. Melt encapsulation at elevated temperatures.

5. Waste disk removal and reactor reset for next cycle. 


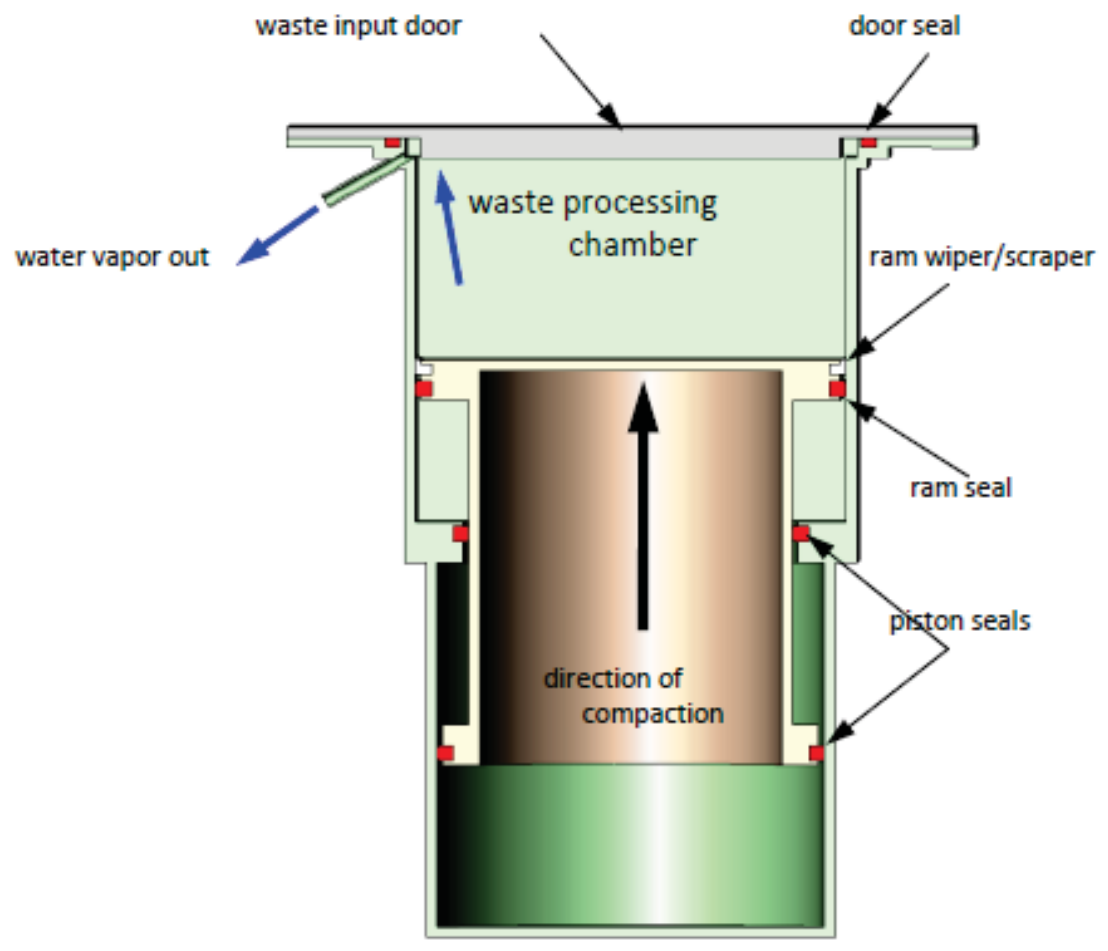

Figure 1. Cut-away view of the HMC design concept.

Phase A needs to be investigated by performing reduced gravity flight tests. In this paper, we develop analytical models to determine the drying time and water recovery rate from the HMC for Phase B during reduced gravity operation and compare these results with ground test data.

\section{Model of Water Recovery Rate}

During Phase B of the HMC process, the heated surfaces are raised to temperatures above the saturation temperature of water but below the melting temperature of the plastic in the trash. During this phase the waste chamber may be exposed to different selected pressures such as ambient, low pressure (e.g., $0.2 \mathrm{~atm}$ ), or vacuum. The objective is to remove both bound water and any remaining free water by evaporation. Keeping the temperature below the plastics melting temperature avoids encapsulation of wet trash that may block vapor escape and reduce the net water recovered.

\section{A. Analysis Objective}

The overall objective of the analysis is to determine the drying time and water recovery rate from the HMC during reduced gravity operation. A second major objective is to determine the trash temperature as the evaporation process proceeds. It may be expected that higher wall temperatures will hasten the evaporation process, but wall temperatures must be kept below the melting temperature of the plastic constituents to avoid trash encapsulation. However, if the bulk of the trash remains much cooler than the wall, it may be possible to raise the wall temperatures to increase evaporation rates.

\section{B. Model Description}

During Phase A, the trash undergoes substantial compaction; only minimal additional compaction takes place during heating of the trash and water evaporation in Phase B. With the heaters maintained at $\sim 120^{\circ} \mathrm{C}$, safely below the plastic melt point, plastic constituents may soften somewhat but do not cause any encapsulation that would hinder vapor escape. Similarly, ground-based experiments suggest that the vapor escape path 
is not obstructed when the trash is compressed. In some instances, however, it has been noted that the compacting piston retreats due to build up of some type of resistance in the waste chamber. When this occurs the measured chamber pressure is still close to the set pressure of evacuation (which may be as low as $0.2 \mathrm{~atm}$ ). This suggests that the resistance causing the piston to retreat is not due to excessive build up of vapor pressure from rapidly evaporating water. It has been suggested that water or liquids trapped in pouches or containers present in the chamber evaporate and build up local pressure inside these pouches which resists the compression force of the piston. It is noticed that after some time the piston advances again presumably as the vapor inside the pouches finally escapes. Events such as these are very dependent on the trash sample and ultimately do not appear to impact the overall evaporation rate to any significant degree.

To date, there have been limited studies of heat transfer properties of the trash. In particular, questions on the degree of trash heterogeneity, thermal conductivity and diffusivity variation, and effects of trash wetness on these properties, as well as vapor path descriptions still exist. There is also uncertainty in the spatial distribution of the water content in the trash which will likely be very sample dependent. Taking these uncertainties into account, the modeling activity will proceed from idealized cases such as uniform 1-D trash configurations to more realistic ones as available information increases and/or more detailed predictions are needed.

Therefore, the initial model described here in this paper is a 1-D configuration with spatial variations in the direction of piston compaction alone; radial or transverse variations will be ignored. The methodology pursued in the model will be similar to the classical Stefan problem where a phase-change front is tracked.

Two situations will be modeled:

Case 1. A pure one-dimensional case where heating is provided only at the two end-faces of the trash as shown in Fig. 2(a) and

Case 2. A quasi-one-dimensional case where side-wall heating is accounted for as shown in Fig. 2(b). In both cases the axial extent (denoted as $\mathrm{H}$ in Fig. 2) of the trash is assumed constant during the process. The cross-section of the trash may be a square (or rectangle) or a circle without loss of generality for Case 1. For Case 2 a circular cross-section has been assumed but the overall results are expected to hold for square or rectangular cross-sections as well.

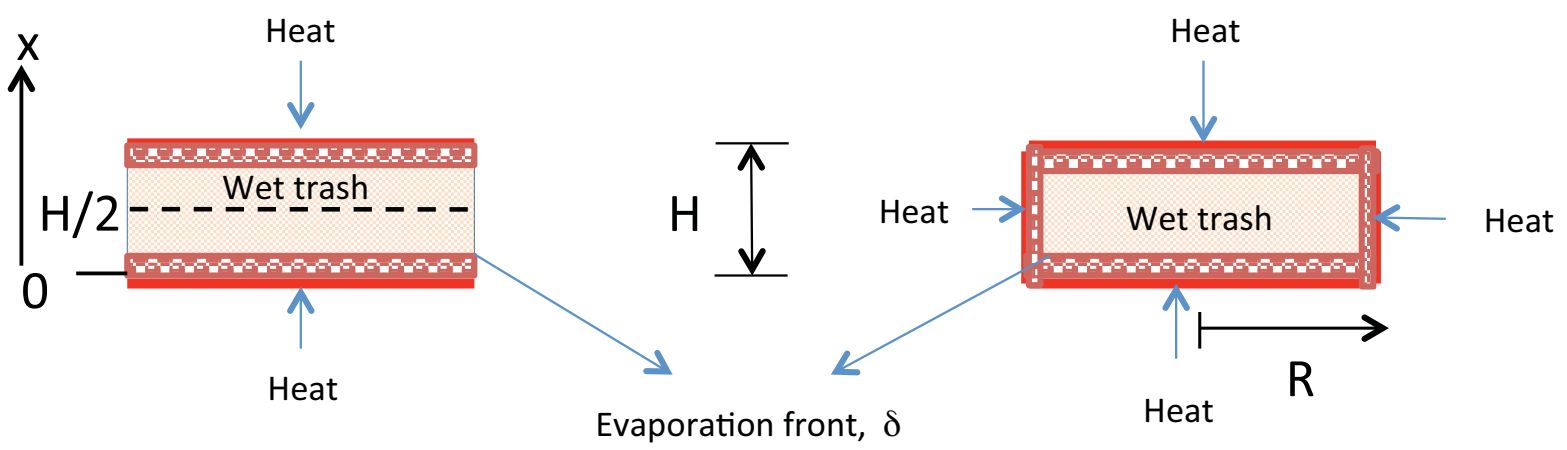

(a)

(b)

Figure 2. (a) One-dimensional case with heating at the two end-faces (b) Quasi-one-dimensional case with inclusion of side-wall heating.

\section{Case 1: 1-D model}

The evaporation front moves from the dry trash (named Region 1) into the wet trash (named Region 2) along the x-axis. Corresponding properties (such as conductivity and density) will be denoted by the appropriate subscript. Consider the energy equation for the trash written as:

$$
\rho_{i} C_{i} \frac{\partial T_{i}}{\partial t}=\frac{\partial}{\partial x}\left(k_{i} \frac{\partial T_{i}}{\partial x}\right)
$$

where $i=1,2$. In this 1 -D analysis, appropriately defined average or effective values of the thermophysical properties are assumed to be known and will be considered as uniform in their respective regions. Statistical 
distributions of these properties are being obtained through testing at NASA ARC and NASA JSC and may be incorporated in the model at a later date. No forced or buoyant convective effects are included in the model. It is assumed that all vapor produced escapes from the trash without blockage, and the chamber pressure is maintained to be constant.

It may be noted that Eq. (1) ignores heat transfer between the trash and the vapor phase. Experiments on the ground have shown that the vapor production rate is about $0.03 \mathrm{~g} / \mathrm{sec}$ from a trash mass of about $500 \mathrm{~g}$. If there is no appreciable build-up of vapor in the waste chamber then the mass of the trash is much greater than that of the vapor present, justifying the neglect of the trash-vapor heat transfer in the energy equation for the trash. The same reasoning indicates that the vapor will attain the temperature of the trash that it passes through prior to leaving the waste chamber. An alternative point of view is that T in Eq. (1) represents a local temperature in the trash regarded as a porous medium, assuming that the various constituents of the porous trash (i.e., plastic, water, water-vapor, etc.) are locally in thermal equilibrium and are therefore at the same temperature.

The initial and boundary conditions are specified as Initial Condition:

$$
T=T(x, t=0) \text { for all } x
$$

Boundary Conditions:

$$
\begin{gathered}
T=T(x=0, t) i . e ., \text { the wall temperature at } x=0 \\
T=T(x=H, t) \text { i.e., the wall temperature at } x=H
\end{gathered}
$$

Interface conditions are also required at the evaporation front $x=\delta(t)$. The first condition is continuity of temperature between Regions 1 and 2 which is expressed as

$$
T_{i}=T_{s}
$$

where $T_{s}$ is the vaporization temperature $(\mathrm{K})$ at the prevailing chamber pressure. The second condition balances the heat fluxes from/to Regions 1 and 2 at the interface.

$$
-k_{1} \frac{\partial T_{1}}{\partial x}=-k_{2} \frac{\partial T_{2}}{\partial x}+\epsilon \rho_{w} \hat{L} \frac{d \delta}{d t}
$$

Note that in general there will be two evaporation fronts, one emanating from each heated end-wall (i.e., $x=0, H)$.

When solving the above system of equations numerically the interface condition is often replaced by a temperature dependent specific heat that accounts for latent heat of vaporization in the vicinity of the evaporation temperature. The description of the specific heat follows Muhieddine et $\mathrm{al}^{1}$ and is given by

$$
C=C_{2}+\left(C_{1}-C_{2}\right) \phi+\hat{L} \frac{d \phi}{d T}
$$

where $\phi=U\left(T-T_{s}\right)$ is the vapor quality and $U$ is the Heaviside unit-step function (whose value is zero for $T<T_{s}$ and unity otherwise), and its derivative, the Dirac delta function is approximated by

$$
\frac{d \phi}{d T}=\frac{\sigma}{\sqrt{\pi}} \exp \left(-\sigma^{2}\left(T-T_{s}\right)^{2}\right)
$$

where $\sigma=\frac{1}{\sqrt{2} \Delta T}$ and $\Delta T$ is a small temperature interval around $T_{s}$.

The numerical solution approach will be most useful when detailed properties of the trash matrix and spatial distribution of water in the trash matrix are known. For example, if the trash is arranged in layers with varying water composition the numerical model will then be able to account for these variations without employing the assumption of homogeneous properties. Similarly, model predictions for a few cases with different assumed spatial distributions of water in the trash matrix are given below. The numerical approach is also useful when side-wall temperatures are varied with time throughout the evaporation process (possibly for improved control). For example, in a practical case, heater cycling may be employed with a PID control system. The numerical approach can incorporate different time-dependent temperature profiles to simulate these conditions. 
It is also useful to obtain analytical solutions of the evaporation process to identify parameters of importance and obtain mathematical expressions of their influence on the evaporation time. This requires some further simplification but the assumptions made do not take away from the general application of the solution. The primary assumption made will be that the vaporization process is quasi-steady. This assumption is justified when the motion of the vaporization front is so slow that temperatures in both the wet and dry trash regions have essentially adjusted to the location of the evaporation front before any appreciable evaporation has taken place at that location. This assumption is often utilized in the literature and may be formally justified by comparing the time-scales of the processes. This assumption allows Eq. (1) to be simplified to a steady diffusion equation given by

$$
\frac{d^{2} T_{i}}{d x^{2}}=0
$$

A second assumption made is that the wall temperatures at $x=0$ and $x=H$ are constant and equal, i.e.,

$$
T(t, x=0)=T(t, x=H)=T_{w}
$$

The constant end-wall temperature assumption means that the initial heating of the end-walls to the desired process temperature is ignored. This assumption holds formally when the wall heat-up period is small compared to the overall evaporation time and is consistent with the quasi-steady approximation. The assumption of equal wall temperatures at the two ends is primarily for algebraic convenience as it allows for the application of symmetry at $x=H / 2$ so that only one evaporation front needs to be tracked. Thus, the boundary condition at $x=H$ is replaced by the following symmetry condition.

$$
\frac{d T_{2}}{d x}=0 \text { at } x=H / 2
$$

The numerical and analytical solutions are discussed in Section III.

\section{Case 2: Quasi-1-D Model with Side-Wall Heating}

Case 1 accounts for heating at the end-walls at $x=0$ and $x=H$. In general, however, the side walls of the waste chamber will also be heated. This heating is accounted for in Case 2 in a quasi-1-D fashion. This is done by considering the energy equation for the cross-sectional average of the trash temperature. Formally, this is done by area integration of the 2-D or 3-D energy equation over the directions orthogonal to the $\mathrm{x}$-axis and averaging by dividing the result by the cross-sectional area. Under the same assumptions as in Case 1 , the result for an axisymmetric quasi-steady situation is

$$
\frac{\partial^{2} T_{i}}{\partial x^{2}}+\frac{2}{R}\left(\frac{\partial T_{i}}{\partial r}\right)_{r=R}=0
$$

where $T_{i}$ is now understood to be the cross-section-averaged temperature. The second term in Eq. (9) is related to the heat flux at the side-wall. Consider the case where the side-wall temperature is held constant as in the experiments and assume that this temperature is the same as the end-wall temperature $T_{w}$. Then the following approximation may be used

$$
\left(\frac{\partial T_{i}}{\partial r}\right)_{r=R} \approx \frac{T_{w}-T_{i}}{R}
$$

The same end boundary and interface conditions as in Case 1 apply to Case 2 as well. The solution for Case 2 is also discussed in Section III.

\section{Results and Discussion}

\section{A. Case 1: Numerical solution}

An example solution set for a Case 1 problem is next discussed. The solution presented here was coded in MATLAB. It may be convenient to code more complicated scenarios with two- and three-dimensional dependencies in a multi-physics solver such as COMSOL in the future. 
In the example simulation, the two end heating surfaces are raised to their final temperature (393 K) over a period of approximately 500 seconds as shown in Fig. 3. These surfaces and the trash complex are initially at $293 \mathrm{~K}$. Calculated temperature profiles in the trash complex at various times are shown in Fig. 4. The profiles are symmetric about $x=H / 2$ because of the assumed symmetry of end-wall conditions. For the results shown in Fig 4., we have used $\sigma=1 / \sqrt{2} \mathrm{~K}^{-1}$ and assumed the thermophysical properties of wet and dry trash to be the same. The grid spacing in the $x$ direction in the numerical solution is $0.1 \mathrm{~mm}$ and is sufficient to resolve the liquid-vapor interface. The following suggested values for the various parameters corresponding to tests conducted under 1-g conditions are used (from Pace et al ${ }^{2}, \mathrm{Pace}^{3}$ and Hong $^{4}$ ):
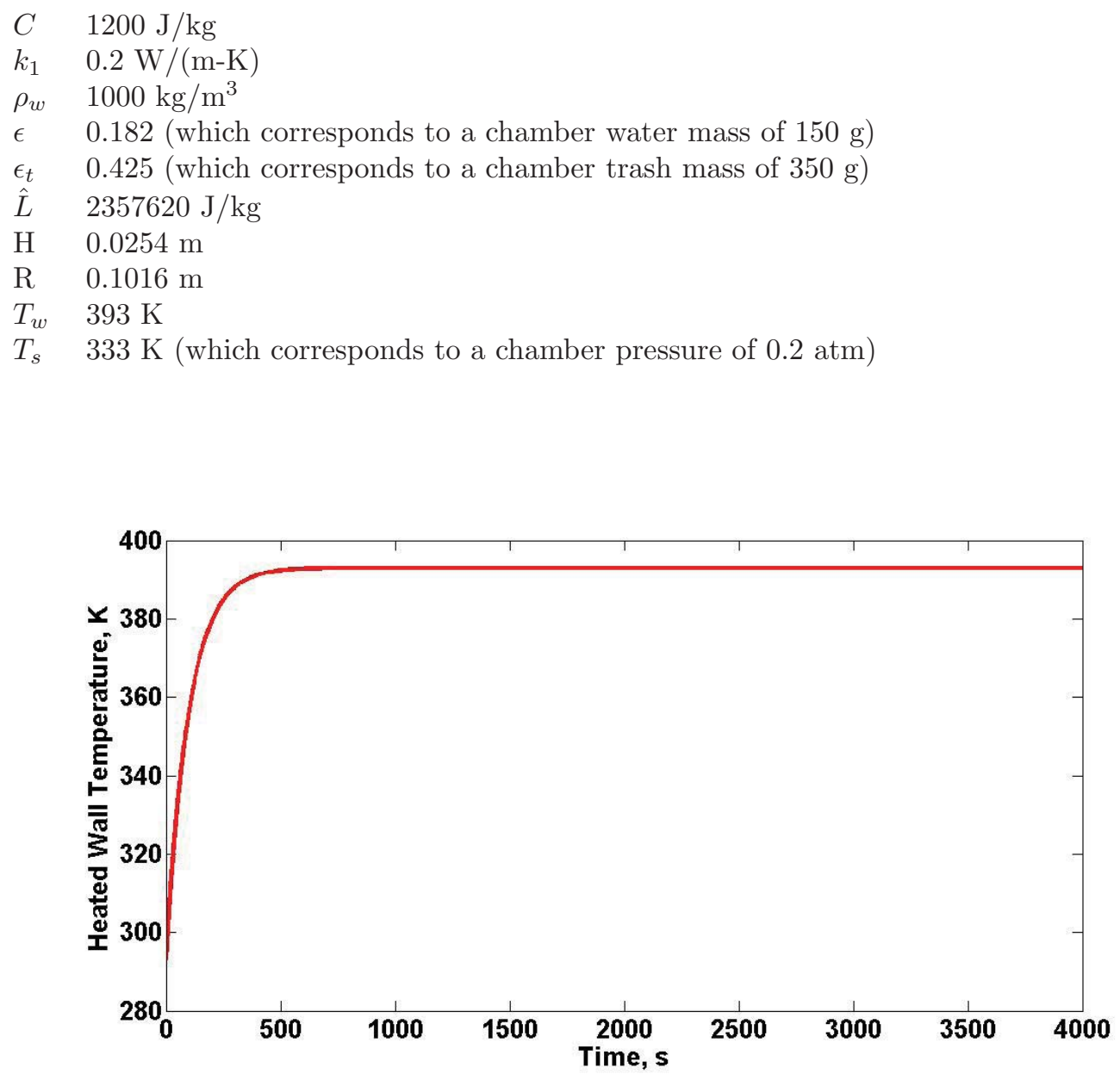

Figure 3. Assumed temperature-time profile of the heated walls. 


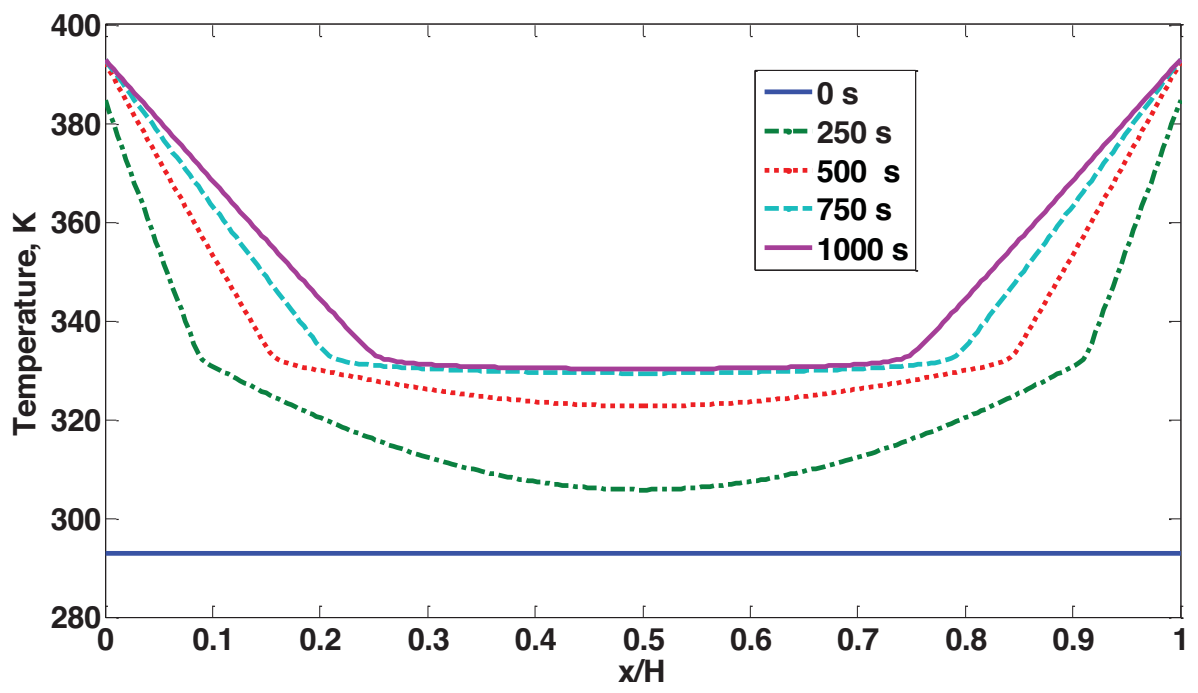

Figure 4. Calculated transient temperature profiles in the trash complex at various times.

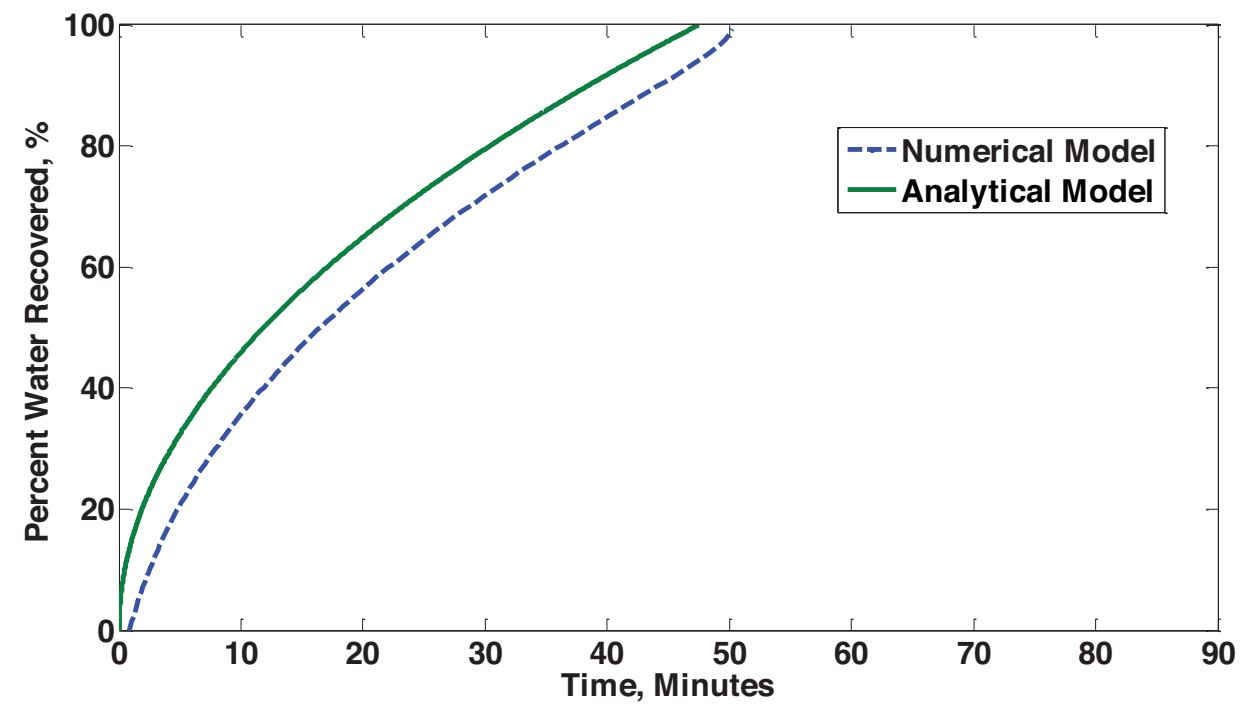

Figure 5. Comparison of numerical and analytical model predictions of water recovered with time. 


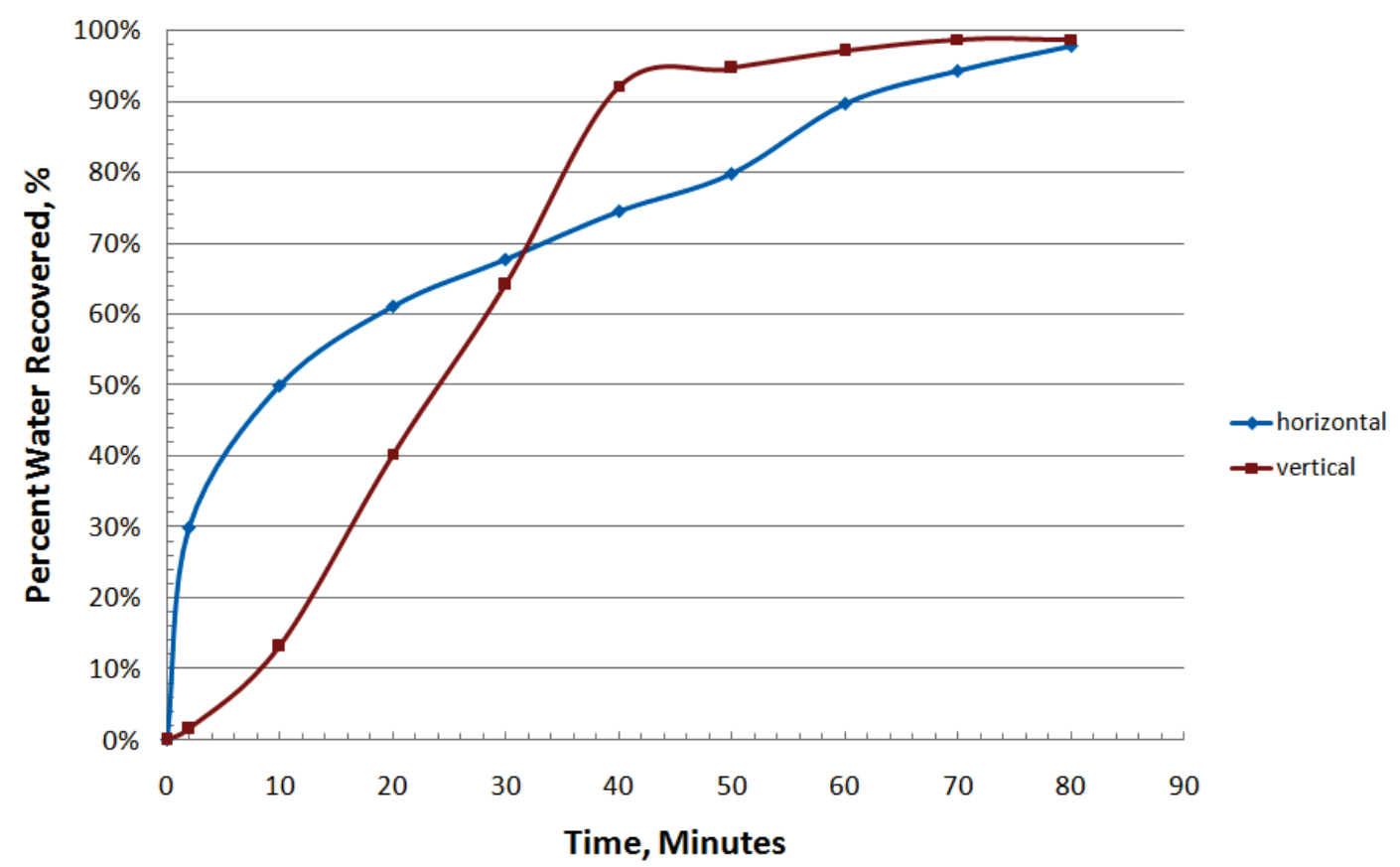

Figure 6. Experimental water recovered with time for horizontal and vertical configurations (from Pace et $\mathrm{al}^{1}$.)

During the transient phase of heating, evaporation commences as soon as the temperature of the endwalls exceeds the vaporization temperature (assumed to be $333 \mathrm{~K}$, i.e., $60 \mathrm{C}$ ) at the operational chamber pressure (assumed to be $0.2 \mathrm{~atm}$ ). The progress of the vaporization front may be tracked by following the kink in the temperature profiles. Note that the temperature profiles become flat in the wet trash once the transient heating of the end-walls is complete. Thus, there is a linear temperature variation from the wall temperature to the vaporization temperature at the vaporization front in the dry trash region and a constant temperature equal to the vaporization temperature in the wet trash region. It will be shown below that this is consistent with the analytical model.

\section{B. Case 1: Analytical solution}

The analytical solutions are as follows:

Region 1 (Dry trash: $0<x<\delta$ )

$$
T_{1}=T_{w}-\frac{\left(T_{w}-T_{s}\right)}{\delta} x
$$

Thus, in the dry trash region, the temperature decreases linearly from the hot-wall temperature $T_{w}$ to the temperature $T_{s}$ at the evaporation front.

Region 2 (Wet trash, $\delta<x<H / 2$ )

$$
T_{2}=T_{s}
$$

Thus, in the wet trash region, the temperature is constant at the temperature $T_{s}$ pending the arrival of the evaporation front. Note that in the quasi-steady approximation, the sensible heating of the wet trash from its initial temperature to the saturation temperature is accomplished much earlier than the time of arrival of the evaporation front.

The solutions for Regions 1 and 2 as shown above may be compared to the numerically computed temperature profile trends in Fig. 4 which shows the same behavior once the transient heating of the walls to their final temperatures is complete. 
The interface evaporation condition (Eq. (3)) yields the following differential equation for the evaporation front location $\delta$ :

$$
\epsilon \rho_{w} \hat{L} \frac{d \delta}{d t}=k_{1} \frac{\left(T_{w}-T_{s}\right)}{\delta}
$$

Integration of the above equation provides $\delta$ as a function of time

$$
\delta=\sqrt{\frac{2 k_{1}\left(T_{w}-T_{s}\right) t}{\epsilon \rho_{w} \hat{L}}}
$$

Therefore, the location of the evaporation front is proportional to the square root of time.

Under the assumptions of the model, the cumulative mass fraction of water, M, evaporated from the trash (relative to the total initial mass of water in the trash) is twice that evaporated from the half region $0<x<H / 2$ and is given by the expression

$$
M=\frac{4}{H} \sqrt{\frac{k_{1}\left(T_{w}-T_{s}\right) t}{2 \epsilon \rho_{w} \hat{L}}}
$$

The calculated time variation of the cumulative water out from the numerical model is compared with the analytical model result in Fig. 5. Except for a small shift in time which accounts for the heat-up of the end-walls in the numerical model both cases are similar and exhibit the square-root dependence on time. This finding gives confidence that the evaporation rate calculated by the analytical model provides the right dependencies on the trash parameters. The overall time for evaporation, $t_{d r y}$, is obtained by setting $\delta=H / 2$. This yields

$$
t_{d r y}=\frac{\epsilon \rho_{w} \hat{L} H^{2}}{8 k_{1}\left(T_{w}-T_{s}\right)}
$$

Noting that the mass of water in the trash is $M_{w}=\pi \epsilon \rho_{w} R^{2} H$, we may write

$$
t_{d r y}=\frac{M_{w} \hat{L} H}{8 \pi R^{2} k_{1}\left(T_{w}-T_{s}\right)}
$$

Equations (16) and (17) for the drying time are the primary result for the 1-D analysis described in this paper. With the values for the trash complex properties given earlier, the time for evaporation is calculated from Eq. (16) as $t_{d r y}=2852 \mathrm{~s}$. While this time is very comparable to experiments conducted in both horizontal and vertical orientations of the HMC (see Fig. 6), the behavior of the cumulative water output curve corresponds better with the horizontal orientation case. The horizontal test result does appear to exhibit a behavior similar to a square-root time dependence; however, the vertical test result appears to be more piece-wise linear in time. It has been surmised that in the vertical case liquid water may have been discharged into the annulus between the waste chamber wall and the outside wall and evaporated there via boiling. However, further investigation of this problem would likely require some repeat testing.

It is instructive to consider the factors that influence the drying time according to Eq. (16) or (17). When the volume fraction $\epsilon$ is specified, the drying time is proportional to $\epsilon$ and to the square of the axial height, $\mathrm{H}$, of the compressed trash. Reducing $\mathrm{H}$ to the extent possible, i.e., without impeding the flow of vapor from the trash complex, will be beneficial to reducing the drying time. When the mass of water in the trash is specified, the drying time is linearly proportional to $M_{w}$ and $\mathrm{H}$. The drying time is inversely proportional to the thermal conductivity of the dry trash, $k_{1}$, and the difference between the wall and vaporization temperatures. While the wall and vaporization temperatures are controllable, the value of the thermal conductivity needs to be experimentally determined. Knowledge of the statistical distribution of $\mathrm{k}$ will be useful for making further predictions. It is clear from Eq. (16) that a given uncertainty in k will translate to a corresponding uncertainty in the drying time. Recent work at JSC to measure the thermal conductivity of trash after melting and resolidification indicates values for conductivity for the dry trash in the range 0.11 to $0.15 \mathrm{~W} / \mathrm{m}-\mathrm{K}$ at $T=20^{\circ} \mathrm{C}$ and 0.14 to $0.165 \mathrm{~W} / \mathrm{m}-\mathrm{K}$ at $T=50^{\circ} \mathrm{C}$ for various samples $\left(\right.$ Hong $\left.^{4}\right)$. We have used $k_{1}=0.2 \mathrm{~W} / \mathrm{m}-\mathrm{K}$ in our calculations. For example, a 20 percent lower conductivity (i.e., $0.16 \mathrm{~W} / \mathrm{m}-\mathrm{K}$ vs $0.2 \mathrm{~W} / \mathrm{m}-\mathrm{K}$ as utilized above) yields a 25 percent higher drying time (i.e., $3565 \mathrm{~s}$ vs $2852 \mathrm{~s}$ ) with all other parameters the same.

It appears, given the agreement between model and experimental results, that the neglect of convective effects in the model is acceptable for predicting the experimental results. It will be useful to confirm this with 
additional model comparisons with experimental results as they become available. If the model is validated, an important conclusion may be made that if the trash complex properties are not significantly different in microgravity then the drying time in microgravity will be of the same order as in $1-\mathrm{g}$ on the ground.

\section{Case 1: Effect of non-uniform water distribution}

The numerical and analytical results presented above assume that $\epsilon$, the volume fraction of water in the trash is initially spatially uniform. In reality, this assumption may not be valid. Each batch of trash loaded into the HMC is likely to have variations in both the amount of water it contains and also how the water is distributed within the trash, depending on how the individual water-containing components of the trash accumulated.

Consider, for example, a sinusoidally varying initial water volume fraction given by the expression $\epsilon(x)=$ $\frac{1}{2} \bar{\epsilon} \pi \sin \frac{\pi x}{H}$. The water distribution is zero at the two ends of the trash $(x=0, \pi)$ and peaks in the middle. $\bar{\epsilon}$ is the mean water volume fraction in the trash. The time-evolution of the location of the liquid/vapor interface may be determined by using $\epsilon(x)$ given above in Eq (13). Defining $\bar{\delta}=\frac{\delta}{H}$ and $\bar{t}=\frac{t}{\tau}$ where $\tau=\frac{\bar{\epsilon} \rho_{w} \hat{L} H^{2}}{2 k_{1}\left(T_{w}-T_{s}\right)}$, the equation for $\bar{\delta}$ may be written as

$$
\bar{\delta} \frac{d \bar{\delta}}{d \bar{t}}=\frac{1}{\pi \sin (\pi \bar{\delta})}
$$

with the initial condition $\bar{\delta}=0$ at $\bar{t}=0$. The drying time is determined as the time when $\bar{\delta}=\frac{1}{2}$. Numerical calculations show that the scaled drying time is $\bar{t}_{d r y}=0.318$, and the physical drying time is $t_{d r y}=3631$ $\mathrm{s}$ for parameter values as in the case of uniform water distribution (for which the drying time is $2852 \mathrm{~s}$ ). We see that the drying time is longer for the sinusoidal water distribution than that for a uniform water distribution. The reason may be explained as follows. In the sinusoidal case, there is more water that is initially present away from the heaters than near the heater surfaces. Since the heat flux from the heater is equal to $\frac{k_{1}\left(T_{w}-T_{s}\right)}{\delta}$, we see that the heat flux is reduced as $\delta$ (and consequently the distance from the heater) increases. Thus, for a sinusoidal water distribution, more heat is required to vaporize the water precisely when the heat flux is low. As a result, the time taken to evaporate the water increases.

The explanation described above enables us to determine the minimum and maximum drying time for a given amount of water in the trash, when the water volume fraction distribution is unknown, but restricted to be one-dimensional. The minimum drying time would occur when all the water is contiguous and is located closest to the heater. Since our analysis is based on two heaters at either ends of the trash, the minimum drying time would occur when equal amounts of water $\left(\frac{1}{2} \epsilon \pi R^{2} H\right)$ occupy a layer of thickness $\frac{\epsilon H}{2\left(1-\epsilon_{t}\right)}$ next to the heaters, where $\epsilon_{t}$ is the trash volume fraction (assumed to be spatially constant). The time evolution of the location of the liquid/vapor interface is as described in Eq (13). The interface is at $x=0$ at $t=0$ and moves to $x=\frac{\epsilon H}{2\left(1-\epsilon_{t}\right)}$ at the time the trash is totally dry. The drying time can be calculated to be

$$
t_{d r y, \min }=\frac{\epsilon^{2} H^{2} \rho_{w} \hat{L}}{8 k_{1}\left(1-\epsilon_{t}\right)\left(T_{w}-T_{s}\right)}=\frac{\epsilon M_{w} \hat{L} H}{8 \pi R^{2} k_{1}\left(1-\epsilon_{t}\right)\left(T_{w}-T_{s}\right)}=\frac{\epsilon}{1-\epsilon_{t}} t_{d r y}
$$

Thus the minimum drying time is smaller than the drying time when the water distribution is uniform (Eq 16) by a factor of $\frac{\epsilon}{1-\epsilon_{t}}$.

The maximum drying time can be similarly determined. It occurs when water is present as a contiguous layer of thickness $\epsilon H$ midway between the heaters. The maximum drying time can be obtained to be

$$
t_{d r y, \max }=\frac{\epsilon\left(2-\epsilon-2 \epsilon_{t}\right) H^{2} \rho_{w} \hat{L}}{8 k_{1}\left(1-\epsilon_{t}\right)\left(T_{w}-T_{s}\right)}=\frac{\left(2-\epsilon-2 \epsilon_{t}\right) M_{w} \hat{L} H}{8 \pi R^{2} k_{1}\left(1-\epsilon_{t}\right)\left(T_{w}-T_{s}\right)}=\left(\frac{2-\epsilon-2 \epsilon_{t}}{\epsilon}\right) t_{d r y, \min }
$$

For the values of the parameters used to calculate the drying time for a uniform water distribution (for which $t_{d r y}=2852 \mathrm{~s}$ ), the minimum and maximum drying times are obtained to be $914 \mathrm{~s}$ and $4857 \mathrm{~s}$, respectively. The minimum drying time is somewhat larger than the initial heat-up time to establish quasisteady temperature profiles in the trash. The ratio of maximum to minimum drying time is $\frac{2-\epsilon-2 \epsilon_{t}}{\epsilon}=5.3$ for $\epsilon=0.182$ and $\epsilon_{t}=0.425$. For low water content, this ratio can be quite large. Note that for the above estimates (especially for the maximum drying time) we are restricted to a strictly 1-D distribution of water, because the theory we have developed is only applicable in this situation. In reality, the water distribution in 
the trash will be three-dimensional, and the maximum drying time will likely be different than that predicted above.

Since a sphere encloses a maximum volume of water to be evaporated with minimum surface area available for heating, we anticipate that a spherical wet region in the trash of an appropriate size might yield the maximum drying time. The drying time for a spherical wet region with an initial diameter $D_{0}$ that is embedded in the trash may be determined as follows. Assume that the trash is heated uniformly to a temperature $T_{w}$ by the heaters. To easily calculate the rate of heat transfer to the sphere, we assume that the trash is of infinite extent. Under the quasi-steady assumption, the spherical wet region will be at a uniform temperature $T_{s}$, and the temperature profile in the vicinity of the wet region in conductive heat transfer is spherically symmetric and is given by

$$
T(r)=T_{w}-\left(T_{w}-T_{s}\right) \frac{D}{2 r}
$$

where $r$ is the radial coordinate from the center of the sphere and $D$ is the instantaneous diameter of the wet region. Noting that the volume fraction of water in the wet region is $1-\epsilon_{t}$, the instantaneous diameter can be calculated by an overall energy balance for the sphere

$$
\begin{gathered}
-\pi D^{2} k_{1}\left(\frac{\partial T}{\partial r}\right)_{r=\frac{D}{2}}=\frac{d}{d t}\left[\frac{\pi D^{3}}{6}\left(1-\epsilon_{t}\right) \rho_{w} \hat{L}\right] \\
D^{2}=D_{0}^{2}-\frac{8 k_{1}\left(T_{w}-T_{s}\right) t}{\left(1-\epsilon_{t}\right) \rho_{w} \hat{L}}
\end{gathered}
$$

where $D_{0}$ is the initial diameter of the wet region. The drying time is given by

$$
t_{\text {dry }, \text { sphere }}=\frac{\left(1-\epsilon_{t}\right) \rho_{w} \hat{L} D_{0}^{2}}{8 k_{1}\left(T_{w}-T_{s}\right)}
$$

Since the diameter of the largest spherical wet region must equal the trash height $H$, the maximum drying time is

$$
t_{d r y, \text { max }, \text { sphere }}=\frac{\left(1-\epsilon_{t}\right) \rho_{w} \hat{L} H^{2}}{8 k_{1}\left(T_{w}-T_{s}\right)}
$$

and is $9110.4 \mathrm{~s}$ for $\epsilon_{t}=0.425$, which is nearly twice as large as the maximum drying time form the 1-D theory.

\section{Case 2: Effect of side-wall heating}

For ease of comparison with Case 1 results as well as for algebraic simplicity, the properties in the wet and dry trash regions will be assumed to be the same. Then, the analytical solutions are as follows:

Region 1 (Dry trash, $0<x<\delta$ )

$$
T_{1}=T_{w}-\left(T_{w}-T_{s}\right) \frac{\sinh (a x)}{\sinh (a \delta)}
$$

where $a=\sqrt{2} / R$.

Region 2 (Wet trash, $\delta<x<H / 2$ )

$$
T_{2}=T_{w}-\left(T_{w}-T_{s}\right) \frac{\cosh \left(a\left(\frac{H}{2}-x\right)\right)}{\cosh \left(a\left(\frac{H}{2}-\delta\right)\right)}
$$

The equation for $\delta$ is given by

$$
\int_{0}^{\delta} \sinh (a \delta) \cosh (a(H / 2-\delta)) d \delta=\frac{k_{1} a\left(T_{w}-T_{s}\right) \cosh \left(a \frac{H}{2}\right)}{\epsilon \rho_{w} \hat{L}} t
$$

Noting that the aspect ratio $\frac{H}{2 R}$ is small (i.e. of order 0.1 for cases of interest) compared to unity allows much simplification. Carrying terms up to order $\left(\frac{H}{2 R}\right)^{2}$ yields the following result for $\delta$ which may be compared to the result for Case 1 (see Eq. 14)

$$
\delta=\sqrt{1+\frac{H^{2}}{4 R^{2}}} \sqrt{\frac{2 k_{1}\left(T_{w}-T_{s}\right) t}{\epsilon \rho_{w} \hat{L}}}
$$


In fact, dividing Eq. (29) by Eq. (14) yields the ratio of evaporation front location with and without side-wall heating to be $\sqrt{1+\frac{H^{2}}{4 R^{2}}}$. Thus, side-wall heating only provides a small benefit when the aspect ratio of the compacted trash is small. In general, for all aspect ratios, side-wall heating increases the energy input to the trash and leads to faster propagation of the evaporation front and decreased drying time compared to results without side-wall heating. If the aspect ratio is not small, Eq. (29) will not be valid and the calculations will need to be modified. Note that for any aspect ratio, if the side wall is well insulated, the 1-D analysis of Case 1 holds, and the drying time can be calculated from Eq. (16). Note also that the side-wall heating does not affect the square root dependence on time for small aspect ratio geometries as shown by Eq. (29).

\section{Conclusion}

We have developed a model for the drying of wet trash in a heat melt compactor neglecting the effects of convective heat transfer. The model predictions and trends are in reasonable agreement with results from tests conducted in normal gravity. The model is intended, however, to predict the drying time in reduced gravity. In the absence of convective effects, the model results do not explicitly depend upon the gravity level. Gravity effects can appear implicitly due to differences in trash properties, chiefly controlled by variations in the water spatial distribution within the trash. When the movement of the vaporization front in the trash is slow compared with thermal conduction time scales, explicit expressions have been derived that relate the water recovery rate and the drying time to the geometric and process operating parameters. The onedimensional model predictions show that the drying time is chiefly controlled by the thermal conductivity of the dry trash, which would be independent of the gravity level, and the distribution of the water volume fraction, which may be highly g-level dependent. Variations in the water distribution, which likely depend on how the trash is mixed and loaded into the HMC, can result in large differences in the drying time, especially for low water content. The model demonstrates that loading the wetter trash closer to the heater ends of HMC and having relatively drier trash in the central portion would help reducing the drying time. Sidewall-heating of the trash is shown to have only a small benefit when the length to diameter ratio of the compacted trash is a small quantity. These results are expected to be generalized to 2-D and 3-D situations as well.

\section{References}

\footnotetext{
${ }^{1}$ Muhieddine, M., Canot, E., and March, R., Various Approaches for Solving Problems in Heat Conduction with Phase Change, International Journal of Finite Volume Method, Vol. 6, No. 1, 2008.

${ }^{2}$ Pace, G. S., Fisher, J., Delzeit, L., Alba, R., and Polonsky, A., Development of a Plastic Melt Waste Compactor for Human Exploration Missions A Progress Report. AIAA 2010-6010, American Institute of Aeronautics and Astronautics, 2010.

${ }^{3}$ Pace, G., Private Communication: HMC_Waste_Model_Thermal_Properties_Test_8-27-2008_new_cotton_and_air.xls.

${ }^{4}$ Hong, A., Private Communication: Heat Melt Compactor Thermal Conductivity Update, April 2, 2012.
} 


